\title{
Perfect timing: Epigenetic regulation of the circadian clock
}

\author{
Jürgen A. Ripperger ${ }^{\mathrm{a}, *}$, Martha Merrow ${ }^{\mathrm{b}}$

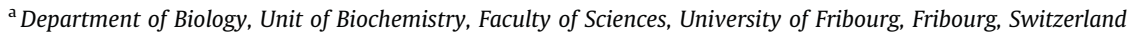 \\ ${ }^{\mathrm{b}}$ Department of Molecular and Genetic Chronobiology, Faculty of Mathematics and Natural Sciences, University of Groningen, Groningen, The Netherlands
}

\begin{abstract}
A B S T R A C T
In mammals, higher order chromatin structures are critical for downsizing the genome (packaging) so that the nucleus can be small. The adjustable density of chromatin also regulates gene expression, thus this post-genetic molecular mechanism is one of the routes by which phenotype is shaped. Phenotypes that arise without a concomitant mutation of the underlying genome are termed epigenetic phenomena. Here we discuss epigenetic phenomena from histone and DNA modification as it pertains to the dynamic regulatory processes of the circadian clock. Epigenetic phenomena certainly explain some regulatory aspects of the mammalian circadian oscillator.
\end{abstract}

\section{Some basic considerations concerning epigenetics and the circadian clock}

Genetic information is encoded in a relatively simple fashion. An average protein of between 50 and $100 \mathrm{kDa}$ requires between 1.35 and $2.7 \times 10^{3}$ nucleotides (nt). If we accept current estimates of circa 30,000 genes in the mammalian genome, then approximately $5 \times 10^{7} \mathrm{nt}$ could represent real protein coding information. This is only about $1-2$ percent of the entire amount of DNA present in a cell. This observation invites two simple hypotheses: the extra DNA must be somehow used for shaping the expression of the genome (else it would not survive evolution since so much extra-genic DNA is energy-expensive to build and maintain) and the vast amount of DNA necessitates an efficient packaging mechanism for considerations of both size and organization. The latter point is facilitated by the formation of nucleosomes, which are DNA wrapped around histone octamers [1]. These can then form higher order structures enabling further compaction of DNA [2-4]. Most of the mammalian genome is packaged as heterochromatin, which is very dense, but regions that are transcriptionally active are less dense and are referred to as euchromatin. As a simple correlation, the tighter the packaging of the local chromatin structure, the less probable it is that transcription and gene expression will occur.

The information on the local chromatin structure can be inherited to the next generation of cells. Thus, the local chromatin structure can exert epigenetic regulation, i.e., it modifies gene

\footnotetext{
* Corresponding author.

E-mail address: Juergenalexandereduard.Ripperger@unifr.ch (J.A. Ripperger).
}

expression and consequently specific phenotypes in a heritable fashion without mutation of the DNA sequence. In mammals, the information reflecting the local chromatin structure is embedded in at least two distinct ways: (i) methylation of specific residues of the local histones [3,5], which are evenly distributed to the newly synthesized DNA during DNA replication, or (ii) methylation of specific cytosine residues in the DNA whose patterns can be enzymatically copied [6,7]. Hence, both kinds of marks can be easily copied to the newly synthesized DNA strands and transferred as such to subsequent generations. Note that temporal regulation of epigenetic modifications was initially not taken into consideration, as these marks were considered highly stable, even over generations. This time scale is obviously not relevant for dynamic processes such as the circadian clock.

Research into the molecular mechanism of circadian oscillators generally focuses on complex genetic networks based on transcriptional/post-translational feedback loops [8,9]. In essence, a gene product accumulates to a concentration upon which it represses its own transcription directly or indirectly via other transcriptional regulators. Consequently, the protein concentration is declining and a following cycle occurs about a day later. Combining these dynamic regulatory systems together with static, long-term epigenetic phenomena is on the first glance counterintuitive. However, recent research suggests that histone and DNA methylation are much more dynamic than previously thought. For instance, over a hundred DNA methylation sites oscillate in synchrony with the cell cycle [10] and the cell cycle is often gated by the circadian clock [11-14], an observation that brings epigenetic regulation into the range of circadian timing. Hence, these processes might play a 
role within the mammalian circadian oscillator as well. They may be fundamental to phenomena like hysteresis of repression at the regulatory regions of circadian target genes [15], tissue-specific gene expression, or passing of oscillator information to daughter cells during mitosis [14].

\section{The histone code of repression: reversible methylation?}

Nucleosomes represent the smallest unit of DNA compaction. There exist about $3 \times 10^{7}$ nucleosomes in the mammalian nucleus. They consist of a histone octamer, which is surrounded by about 1.67 loops of DNA. The 'DNA content' of a nucleosome is about 147 base pairs. Due to the specific surface properties of the histone octamer, DNA is relatively free to move around it [16]. In addition, about 3/4 of the DNA strand is accessible e.g., to transcription factors. Surprisingly, although they are bound to DNA, nucleosomes are not barriers to the general processes of DNA replication or transcription. During replication, the histones $\mathrm{H} 3$ and $\mathrm{H} 4$ are evenly distributed to the new DNA strands [17] and during transcription the DNA can loop out from the nucleosome to allow access to for instance RNA-polymerases [18].

However, nucleosomes can also exert regulatory effects on transcription and therefore gene expression $[19,20]$. This function is mediated by the amino-termini of histones, which protrude and are quite flexible [21]. Many of the amino acid residues in these histone tails are prone to post-translational modification. In general, acetylation of lysine residues or phosphorylation of serine residues is associated with an open chromatin configuration, which facilitates transcription. However, the precise mode of action of the modified histone tails is not known yet. Alternative hypotheses are that the chromatin configuration is either directly affected by the negative charges introduced into the histone tails via changing histone-histone interactions, or because specific proteins recognize and interact with these modifications.

A higher nucleosome density, depletion of acetylation and phosphorylation, or the presence of mono-, di- or tri-methylated lysine residues in the histone tails reflect a repressive transcriptional mode [5]. The latter modifications are specific binding sites for e.g., heterochromatin proteins (Hp) $[22,23]$ or polycomb group (PcG) proteins [24], which assemble the chromatin even tighter. The number and kind of histone modifications on the histone tails manifests the histone code. Specific binding proteins can interpret this histone code as markers for transcriptional activation or repression. Since, as already mentioned, nucleosomes and consequently the histone modifications stay attached to the DNA even during DNA replication, the modification state of nucleosomes can be transferred to the subsequent generations of a cell.

A first hint concerning the involvement of histone modifications in circadian timing was found at the Period1 (Per1) and Period2 (Per2) gene promoters [25]. Activation of transcription at these promoters is mediated by BMAL1 and CLOCK heterodimers [8,9]. PER1 and PER2 participate in the so-called negative limb of the mammalian circadian oscillator where they repress BMAL1- and CLOCKmediated transcriptional activation thus creating a feedback loop. In this manner, PER1 and PER2 are important to establish the typical circa 24-h oscillations of this molecular oscillator. Rhythmic acetylation of lysine 9 of histone H3 at the Period1 (Per1) and Period2 (Per2) gene promoters is observed with the phase of histone acetylation coinciding with the phase of transcriptional activity of both genes [25]. Interestingly, one of the transcriptional activators, CLOCK, interacts with the histone acetyltransferase (HAT) p300, which specifically adds acetyl residues to the lysine 9 of histone $\mathrm{H} 3$. The p300 enzyme increases the transcriptional read-out of a luciferase reporter under the control of the mPer1 promoter [25]. These data suggest that time-of-day specific recruitment of HAT activity leads to rhythmic histone acetylation. Similar experiments showed that CLOCK and also NPAS2 recruit various histone acetyltransferases to the mPer1 promoter in vascular tissues [26].

We now know that CLOCK itself possesses HAT activity [27]. Hence, rhythmic binding of the transcriptional activators BMAL1 and CLOCK can directly affect the acetylation of specific lysine residues of histones nearby their DNA binding sites without the help of other HAT enzymes. In addition, the HAT activity of CLOCK also acetylates its heterodimerization partner BMAL1 [28]. The specific acetylated lysine residue of BMAL1, however, is involved in repression rather than activation. Hence, the definitive impact of the HAT activity of CLOCK on the circadian oscillator is still an unanswered question. Nevertheless, rhythmic acetylation of histones - regardless of the details - is broadly occurring in circadian clocks.

A rhythmic counterpart to HAT activity on circadian genes was identified $[29,30]$ in SIRT1, a histone deacetylase. This enzyme apparently removes acetyl residues not only from the histone tails, effectively decreasing transcriptional activation, but also from the PER2 [29] and BMAL1 protein [30], affecting the activity of these proteins. Furthermore, this enzymatic activity is modulated by the intracellular ratio of $\mathrm{NAD}^{+}$to $\mathrm{NADH} / \mathrm{H}^{+}$, providing a link between the metabolic state of the cell and the circadian clock. Histone deacetylation is also recruited to the regulatory clock network by the interaction of the Cryptochrome1 (CRY1) protein with various histone deacetylases (HDACs) [31], or by the interaction of the circadian nuclear receptor REV-ERB $\alpha$ with NCoR1 to recruit HDAC3 [32]. Taken together, histone acetylation and deacetylation are part of the regulatory processes that generate circadian oscillations in gene expression. From a formal point of view, histone acetylation and deacetylation solely correlate with the state of transcription and are hence too transient to represent epigenetic phenomena.

Going more into the direction of epigenetic gene regulation, PER1 and PER2 associate with WDR40 [33], an adaptor targeting lysine 4 of histone $\mathrm{H} 3$ in conjunction with the histone $\mathrm{H} 3$-specific methyltransferase Set1/Ash2. A knock down of WDR40 in fibroblast cell lines leads to a loss of rhythmic histone methylation and alterations in rhythmic gene expression. In particular, the magnitude of mPer2 expression is reduced. Overall, however, the impact of a knock down of WDR40 is modest with respect to the functioning of the circadian oscillator. An explanation for this discrepancy may be that circadian oscillators are largely resilient to fluctuations of transcription rate [34]. Differences in the level of gene expression may therefore be compensated at the posttranslational level.

Additional histone methyltransferases are associated with circadian regulation. The Enhancer of Zeste 2 (EZH2), a histone H3-specific methyltransferase that tags the lysine 27, co-immunoprecipitates with either CLOCK or BMAL1 [35]. A rhythmic pattern of di-/tri-methylation of this residue occurs at the promoter of the mPer1 but not the mPer2 gene and occurs in anti-phase to transcriptional activity of this gene. In co-transfection assays, EZH2 enzymatic activity enhances (overexpression) or decreases (diminished by RNAi) the repressing activity of CRY proteins on a Per1-luc reporter gene in fibroblast cells. Thus, the role of methylation of lysine 27 of histone H3 was shown to affect expression of the Per1 gene. Similarly, recent findings suggest that the methyltransferase myeloid/lymphoid or Mixed-Leukemia Lineage (MLL) rather than Set $1 \mathrm{~A}$ mediates the methylation of histone $\mathrm{H} 3$ lysine 4 to regulate BMAL1 and CLOCK target genes [36].

The clock-controlled albumin $D$-site binding protein $(D b p)$ gene was established as a model for the analysis of rhythmic histone modifications [37]. Highly dynamic, reversible histone methylations occur at this gene (Fig. 1). Methylation of lysine 4 and acetylation of lysine 9 of histone $\mathrm{H} 3$ are in phase with transcriptional activity of the $D b p$ gene. These histone modifications are observed 


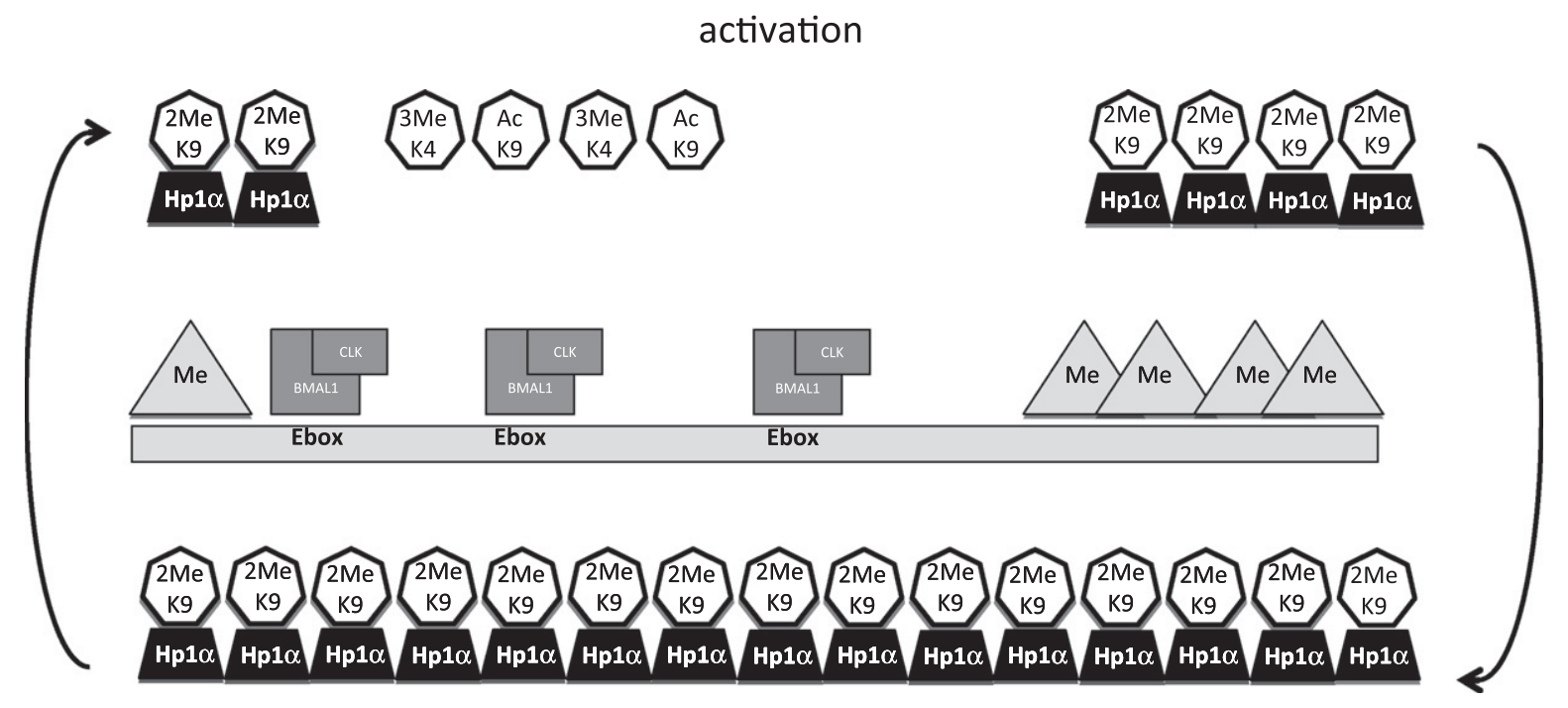

\section{repression}

Fig. 1. Circadian regulation of the mouse Dbp gene. Upon binding of the two transcriptional activators BMAL1 and CLOCK, the local chromatin structure opens to allow transcription. This is accompanied by an increase in the typical markers for active chromatin, tri-methylation of lysine 4 and acetylation of lysine 9 of histone H3 around the promoter site ( 7 sided 'pac-man' units). Eventually, both transcriptional activators fall off their relevant binding sites and acetylation of histones is lost. This effect may be initiated by the recruitment of the SIRT1 histone deacetylase or related enzymatic activities. Finally, the chromatin starts to compact. Markers like di-methylation of lysine 9 of the histone $\mathrm{H} 3$ increase at the promoter region. This allows the spreading of HP1 $\alpha \square \square \square \square \square \square D b p$ gene, which may cause further chromatin compaction. Consequently, a heterochromatin-like state is generated to maintain repression (hysteresis) until a next cycle of transcriptional activation occurs on the following day. Note that stable methylated CpG sites (triangles) flank the Dbp gene, which may represent anchor points for surrounding heterochromatin. Adapted from [37].

prominently at the promoter region and rapidly decrease in $5^{\prime}$ to $3^{\prime}$ direction into the $D b p$ gene. This suggests that both types of modifications are performed by enzymatic activities associated with RNA-polymerase II and that these enzymatic activities dissociate from the elongating RNA-polymerase II during RNA synthesis [38].

At the nadir of $D b p$ gene expression, the picture is completely changed [37]. The above-mentioned histone modifications are strongly reduced and another modification, typical for a repressed state, increases throughout the $D b p$ gene. A di-methylated lysine 9 of histone $\mathrm{H} 3$ - the same residue that is acetylated during activation - becomes a binding site for Heterochromatin protein $1 \alpha$ $(\mathrm{Hp} 1 \alpha)[22,23]$. Consequently, this protein is found during repression all over the $D b p$ gene. In addition, the histone density, as judged by the presence of histone $\mathrm{H} 3$, increases. Altogether, the chromatin at the $D b p$ gene during repression resembles a heterochromatin-like state. Although these features have as yet only been analyzed for the mouse $D b p$ gene, it is tempting to speculate that reversible histone methylation would be part of many circadian regulatory regions.

What is the advantage of reversible histone methylation? The circadian transcriptional activators BMAL1 and CLOCK, and the transcriptional repressors PER1 and PER2 bind only temporarily to the relevant regulatory regions within their target genes. Recent evidence from the Drosophila melanogaster circadian oscillator suggests that the related Per protein is detached together with the analogous activating clock/cycle complex from the DNA [39]. From the kinetics of PER1 and PER2 binding relative to BMAL1-binding, a similar mechanism may be operative in mammals as well [40]. As a consequence, there must be mechanisms that continue the repressive state after the repressors have left the DNA. This concept is referred to as hysteresis [15]. The lysine 9 of histone H3-specific methylation and the binding of $\mathrm{Hp} 1 \alpha$ signal a repressive state even in the absence of circadian repressors. Thus, they maintain the repressive state initiated by transcriptional repressors until a new cycle begins. However, to function properly, all of the histone modifications have to be temporally coordinated (Fig. 2).

\section{Marks on DNA: reversible DNA methylation?}

A very general epigenetic mechanism to inactivate genes is the methylation of cytosines, usually in the context of the sequence motif $\mathrm{CpG}[6,7]$. There are about $5 \times 10^{7}$ potential $\mathrm{CpG}$ sites in the genome. Methylation of these sites either directly interferes with the binding of transcriptional regulators, or indirectly inactivates a gene by initiating the formation of a heterochromatic state. CpG sites within the genomes of mammals are drastically underrepresented (about $20-25 \%$ of their theoretical abundance), and the remaining sites are enriched in functional subunits of the genome (e.g., CpG islands). A recent bioinformatics approach found that the regulatory regions of circadian genes are enriched in CPG motifs relative to other genes [41]. Therefore, an impact of CPG methylation on circadian gene regulation is conceivable.

In mammals, $\mathrm{CpG}$ methylation is performed by three different DNA methyltransferases (DNMTs). The isoforms DNMT3a and DNMT3b perform the de novo methylation of $\mathrm{CpG}$ residues during development, while DNMT1 is supposed to act as a maintenance methyltransferase that is responsible for copying the methylation pattern to the newly synthesized DNA strand during DNA replication [7]. Methylation of $\mathrm{CPG}$ residues provides binding sites for Methyl-CpG-binding proteins (MBPs). Of particular interest are the Methyl-CpG-binding proteins MBP1, MBP2, and MBP3 that have been shown to act as transcriptional repressors by serving as anchor points to recruit components of the repression machinery to a defined locus [42-44]. This machinery, which consists of histone deacetylases and methyltransferases, compacts the DNA around the CPG site into stable heterochromatin [45].

The $\mathrm{CpG}$ methylation status of a cell is epigenetically inherited over many generations. In non-proliferating adult tissues, DNA methylation is considered even more stable than histone methylation patterns in the absence of DNA replication. However, DNA demethylation has been demonstrated to occur in somatic cell nuclei [46] and according to cell cycle stage at some sites [10]. In addition, a publication describes diurnal changes of $\mathrm{CpG}$ methylation in 


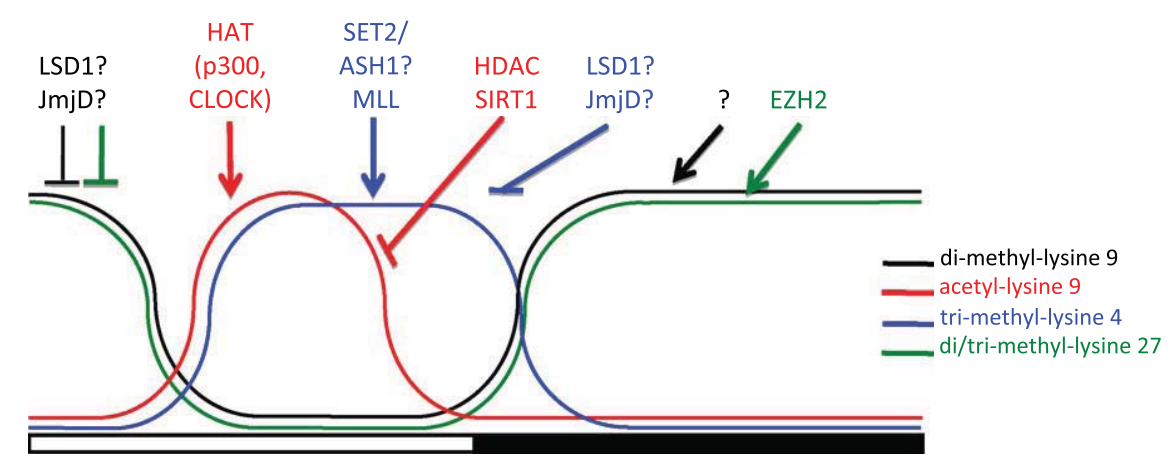

Fig. 2. Model of dynamic chromatin transitions at circadian genes over the $24 \mathrm{~h}$ day (day, open bar at bottom; night, closed bar at bottom). Activation of BMAL1 and CLOCK regulated genes coincides with acetylation of histone residues by HATs, and tri-methylation of lysine 4 of histone H3 by Set2/Ash1 (via a WDR5 adapter) or MLL, and the removal of repressive histone methylations by LSD1 or JmjD-domain proteins. These activities are counterbalanced by the recruitment of HDACs or SIRT1, and LSD1 and JmjDdomain proteins, respectively. Similar scenarios may occur at ROR $\alpha /$ REV-ERB $\alpha$ and DBP regulated circadian target genes as well. Indeed, REV-ERB $\alpha$ was recently identify as the main anchor point for HDAC3 [64].

circulating white blood cells [47]. The authors report a nadir of homocysteine, a degradation product of S-adenosylmethionine, which is the donor of the methyl group transferred to the DNA, and a peak of DNA methylation in the evening in the blood of healthy subjects. The low amplitude of oscillation, which would affect only about $5 \%$ of the total $\mathrm{CpG}$ methylation sites, still translates into a huge number of $\mathrm{CPG}$ sites $\left(2.5 \times 10^{6}\right.$ potential sites). However, to date there is no single $\mathrm{CpG}$ motif known in a mammalian genome that shows circadian oscillations in methylation.

A defined role for $\mathrm{CpG}$ methylation of circadian genes may occur during development. A recent study compared $\mathrm{CpG}$ methylation in the promoter regions of the mPer1, mPer2 and mCry 1 genes. At certain positions within the regulatory region of the mPer1 gene the CpG methylation changes dependent on the developmental state [48]. Interestingly, in vitro methylation within the mPer1 promoter has an inhibitory effect on reporter gene expression. This is a first experimental hint that $\mathrm{CpG}$ methylation could influence circadian rhythms. In addition, one of the strongest associations between the circadian clock and DNA methylation concerns cancerous growth. The expression of many key circadian regulators (e.g., Per1 and Per2) is lost during cancer development. In some cases, restoring expression of circadian clock components prevents malignant growth. $\mathrm{CpG}$ methylation is linked to this phenomenon because of a study that finds inactivation of Per1, Per2 and Cry 1 in endometrial cancers by methylation [49]. Other studies describe hyper-methylation of Per1 in cervical cancer cells [50] or non-small cell lung cancer [51], and of Per3 in chronic myeloid leukemia cells [52], whereas hyper-methylation of sites within the Clock gene reflects a reduced risk of breast cancer development, possibly due to a reduction of the expression of the Clock gene [53].

Taken together, $\mathrm{CpG}$ methylation could exert a function within the mammalian circadian oscillator. Since $\mathrm{CpG}$ methylation is stable, may affect many potential regulatory sites and generates a tight repressive state, it is appealing to speculate that this mechanism provides tissue-specific gene regulation (Fig. 3). Mechanisms that provide reversible methylation of DNA remain to be discovered.
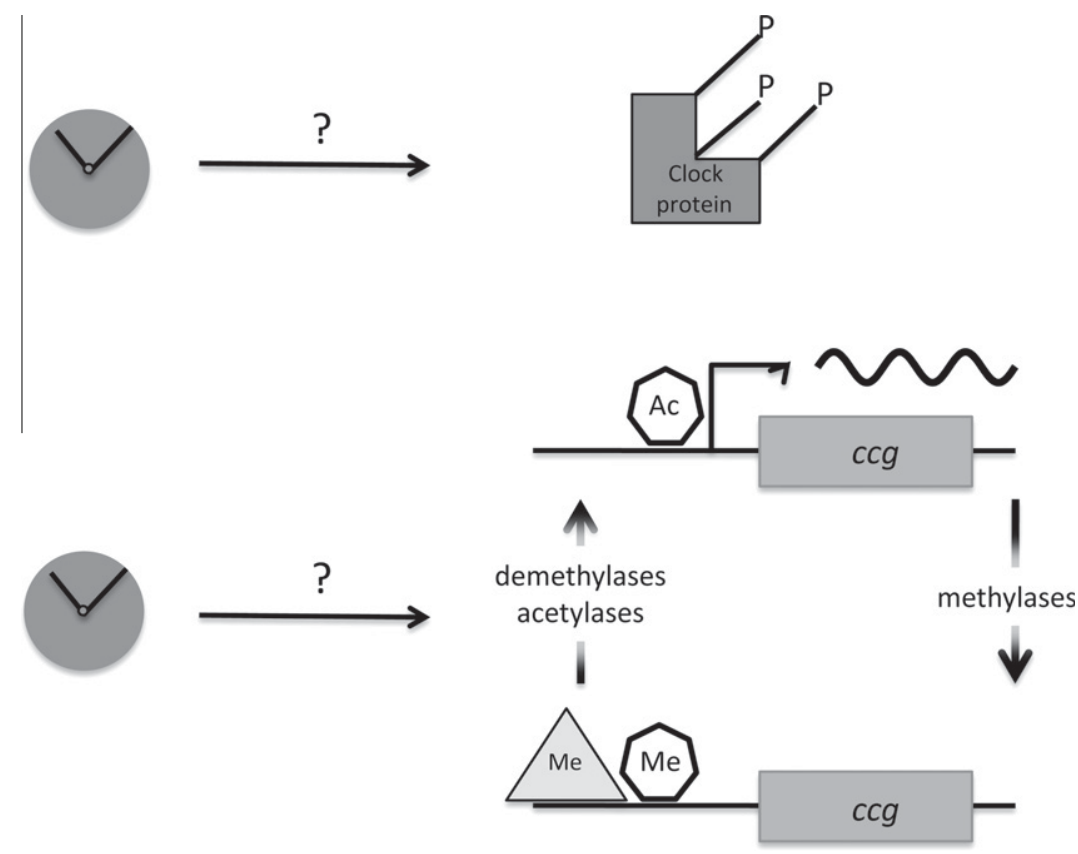

Fig. 3. Propagation of epigenetic information over cellular generations. To transmit temporal information to daughter cells, either circadian oscillator components bearing time-coding post-translational modifications (e.g., phosphorylation, P-P) are evenly distributed to the daughter cells, or methylation patterns (Me) are copied. Changes in the methylation patterns of DNA (triangles) or of the modifications (methylation, Me; acetylation, Ac) on histones allow tissue-specific activation or inactivation of circadian genes. Adapted from [6,7]. 
However, there is no doubt that $\mathrm{CpG}$ methylation impinges on the expression of circadian regulatory genes in cancer cells.

\section{Perspectives}

Until a couple of years ago, histone and DNA methylation were believed to be more or less irreversible. However, numerous enzymatic activities have been identified that remove methyl groups from e.g., specific histone residues. One of the first of these is the lysine specific demethylase 1 (LSD1) [54]. This protein possesses two opposing functions: depending on which complex it is associated with, it can either repress genes by removing the di-methylation of histone $\mathrm{H} 3$ lysine 4 or activate transcription by demethylating lysine 9 [55]. Via specific binding properties, it can be exquisitely targeted to specific genetic loci. Due to its bipartite function, LSD1 is an attractive candidate for a time of day dependent transcriptional regulator. Similarly, another demethylase family is the JmjD-domain containing proteins [56,57]. These demethylases remove tri-methyl groups from lysine residues. The function of these enzymes has been analyzed mainly during the early stages of development, with the JmjD2 sub-family involved in the demethylation of various lysine residues in undifferentiated and differentiated embryonic stem cells [58]. Concerning regulation of posterior development in animals, JMJD3 counterbalances the activity of PcG group proteins with respect to the state of lysine 27 of histone H3 [59]. It acts similarly during inflammation [60] and development [61]. Concerning circadian regulation, JMJD3 may, for instance, directly counteract the activity of EZH2, thereby leading to circadian changes in methylation status of the lysine 27 of histone $\mathrm{H} 3$ at the mPer1 gene. To remove CpG methylation from DNA, Gadd45a has been identified [62]. However, it is currently unclear, whether this protein is a genuine enzyme to remove methyl groups from cytosine, or whether it is involved in a more general DNA repair mechanism. Interestingly, hydroxymethylation of cytosine bases is a quite abundant modification of DNA in the brain [63]. Hydroxymethylation may represent either an independent mark with yet to be specified function(s), or reflect a degradation intermediate of yet to be identified DNA demethylases.

Whether any of these demethylases are expressed in a circadian fashion or involved in circadian regulation remains to be investigated. The opportunities for precise and specific circadian regulation are manifold (Fig. 3). They may acquire specificity via their specific binding partners (e.g., clock transcription factors would bring them rhythmically to the proximity of clock gene promoters or other epigenetic regulatory enzymes) or by the timing of their expression, sub-cellular localization or post-translational modifications. Since many of these processes may be restricted to particular tissues or cell types, it is conceivable that these kinds of phenomena mediate tissue-specific gene expression.

An example of a situation where it would be an advantage to have a memory of circadian time encrypted as an epigenetic mark is cell division (Fig. 3). Experiments in tissue culture cells reveal that identical oscillator information is transferred to daughter cells. The mechanism behind this observation is unclear. Either the components of the circadian oscillator, whose concentrations and secondary modifications reflect circadian time, are evenly distributed to the daughter cells, or epigenetic marks fulfill this task. Clearly, further experiments are required to distinguish between these options.

\section{Acknowledgements}

Our work is supported by the EU FP6 integrated project EUCLOCK (018741), the NWO (the Dutch Science Foundation), the Rosalind Franklin Fellowships of the University of Groningen, and the Swiss National Fonds (SNF/FNS).

\section{References}

[1] Richmond, T.J. and Davey, C.A. (2003) The structure of DNA in the nucleosome core. Nature 423, 145-150.

[2] Dorigo, B., Schalch, T., Kulangara, A., Duda, S., Schroeder, R.R. and Richmond, T.J. (2004) Nucleosome arrays reveal the two-start organization of the chromatin fiber. Science 306, 1571-1573.

[3] Fischle, W., Wang, Y. and Allis, C.D. (2003) Histone and chromatin cross-talk. Curr. Opin. Cell Biol. 15, 172-183.

[4] Schalch, T., Duda, S., Sargent, D.F. and Richmond, T.J. (2005) X-ray structure of a tetranucleosome and its implications for the chromatin fibre. Nature 436, $138-141$.

[5] Kikuchi, H., Barman, H.K., Nakayama, M., Takami, Y. and Nakayama, T. (2006) Participation of histones, histone modifying enzymes and histone chaperones in vertebrate cell functions. Subcell. Biochem. 40, 225-243.

[6] Bird, A.P. and Wolffe, A.P. (1999) Methylation-induced repression - belts, braces and chromatin. Cell 99, 451-454.

[7] Klose, J.R. and Bird, A.P. (2006) Genomic DNA methylation: the mark and its mediators. TIBS 31, 89-97.

[8] Ko, C.H. and Takahashi, J.S. (2006) Molecular components of the mammalian circadian clock. Hum. Mol. Genet. 15, R271-R277.

[9] Young, M.W. and Kay, S.A. (2001) Time zones: a comparative genetics of circadian clocks. Nat. Rev. Genet. 2, 702-715.

[10] Brown, S.E., Fraga, M.F., Weaver, I.C., Berdasco, M. and Szyf, M. (2007) Variations in DNA methylation patterns during the cell cycle of HeLa cells. Epigenetics 2, 54-65.

[11] Carre, I.A. and Edmunds Jr., L.N. (1993) Oscillator control of cell division in Euglena: cyclic AMP oscillations mediate the phasing of the cell division cycle by the circadian clock. J. Cell Sci. 104, 1163-1173.

[12] Homma, K. and Hastings, J.W. (1989) Cell growth kinetics, division asymmetry and volume control at division in the marine dinoflagellate Gonyaulax polyhedra: a model of circadian clock control of the cell cycle. J. Cell Sci. 92, 303-318.

[13] Merrow, M. and Roenneberg, T. (2004) Cellular clocks: coupled circadian and cell division cycles. Curr. Biol. 14, R25-R26.

[14] Nagoshi, E., Saini, C., Bauer, C., Laroche, T., Naef, F. and Schibler, U. (2004) Circadian gene expression in individual fibroblasts: cell-autonomous and selfsustained oscillators pass time to daughter cells. Cell 119, 693-705.

[15] Eissenberg. J.C. and Elgin, S.C. (2006) Marking time. Nat. Genet. 38, 276-277.

[16] Luger, K. and Richmond, T.J. (1998) DNA binding within the nucleosome core. Curr. Opin. Struct. Biol. 8, 33-40.

[17] Xu, M., Long, C., Chen, X., Huang, C., Chen, S. and Zhu, B. (2010) Partitioning of histone $\mathrm{H} 3-\mathrm{H} 4$ tetramers during DNA replication-dependent chromatin assembly. Science 328, 94-98.

[18] Ong, M.S., Richmond, T.J. and Davey, C.A. (2007) DNA stretching and extreme kinking in the nucleosome core. J. Mol. Biol. 368, 1067-1074.

[19] Imhof, A. and Becker, P.B. (2001) Modifications of the histone N-terminal domains. Evidence for an "epigenetic code"? Mol. Biotechnol. 17, 1-13.

[20] Jenuwein, T. and Allis, C.D. (2001) Translating the histone code. Science 293, 1074-1080.

[21] Luger, K. and Richmond, T.J. (1998) The histone tails of the nucleosome. Curr. Opin. Genet. Dev. 8, 140-146.

[22] Bannister, A.J., Zegerman, P., Partridge, J.F., Miska, E.A., Thomas, J.O., Allshire, R.C. and Kouzarides, T. (2001) Selective recognition of methylated lysine 9 on histone H3 by the HP1 chromo domain. Nature 410, 120-124.

[23] Lachner, M., O'Carroll, D., Rea, S., Mechtler, K. and Jenuwein, T. (2001) Methylation of histone H3 lysine 9 creates a binding site for HP1 proteins. Nature 410, 116-120.

[24] Cao, R., Wang, L., Wang, H., Xia, L., Erdjument-Bromage, H., Tempst, P., Jones, R.S. and Zhang, Y. (2002) Role of histone H3 lysine 27 methylation in Polycomb-group silencing. Science 298, 1039-1043.

[25] Etchegaray, J.P., Lee, C., Wade, P.A. and Reppert, S.M. (2003) Rhythmic histone acetylation underlies transcription in the mammalian circadian clock. Nature 421, 177-182.

[26] Curtis, A.M., Seo, S.B., Westgate, E.J., Rudic, R.D., Smyth, E.M., Chakravarti, D., FitzGerald, G.A. and McNamara, P. (2004) Histone acetyltransferasedependent chromatin remodeling and the vascular clock. J. Biol. Chem. 279, 7091-7097.

[27] Doi, M., Hirayama, J. and Sassone-Corsi, P. (2006) Circadian regulator CLOCK is a histone acetyltransferase. Cell 125, 497-508.

[28] Hirayama, J., Sahar, S., Grimaldi, B., Tamaru, T., Takamatsu, K., Nakahata, Y. and Sassone-Corsi, P. (2007) CLOCK-mediated acetylation of BMAL1 controls circadian function. Nature 450, 1086-1090.

[29] Asher, G., Gatfield, D., Stratmann, M., Reinke, H., Dibner, C., Kreppel, F., Mostoslavsky, R., Alt, F.W. and Schibler, U. (2008) SIRT1 regulates circadian clock gene expression through PER2 deacetylation. Cell 134, 317-328.

[30] Nakahata, Y., Kaluzova, M., Grimaldi, B., Sahar, S., Hirayama, J., Chen, D., Guarente, L.P. and Sassone-Corsi, P. (2008) The NAD+-dependent deacetylase SIRT1 modulates CLOCK-mediated chromatin remodeling and circadian control. Cell 134, 329-340.

[31] Naruse, Y., Oh-hashi, K., Iijima, N., Naruse, M., Yoshioka, H. and Tanaka, M. (2004) Circadian and light-induced transcription of clock gene Per1 depends on histone acetylation and deacetylation. Mol. Cell. Biol. 24, 6278-6287. 
[32] Yin, L. and Lazar, M.A. (2005) The orphan nuclear receptor Rev-erbalpha recruits the $\mathrm{N}-\mathrm{CoR} /$ histone deacetylase 3 corepressor to regulate the circadian Bmal1 gene. Mol. Endocrinol. 19, 1452-1459.

[33] Brown, S.A., Ripperger, J., Kadener, S., Fleury-Olela, F., Vilbois, F., Rosbash, M. and Schibler, U. (2005) PERIOD1-associated proteins modulate the negative limb of the mammalian circadian oscillator. Science 308, 693-696.

[34] Dibner, C., Sage, D., Unser, M., Bauer, C., d'Eysmond, T., Naef, F. and Schibler, U. (2009) Circadian gene expression is resilient to large fluctuations in overall transcription rates. EMBO J. 28, 123-134.

[35] Etchegaray, J.P., Yang, X., DeBruyne, J.P., Peters, A.H., Weaver, D.R., Jenuwein, T. and Reppert, S.M. (2006) The polycomb group protein EZH2 is required for mammalian circadian clock function. J. Biol. Chem. 281, 21209-21215.

[36] Katada, S. and Sassone-Corsi, P. (2010) The histone methyltransferase MLL1 permits the oscillation of circadian gene expression. Nat. Struct. Mol. Biol. 17, $1414-1421$.

[37] Ripperger, J.A. and Schibler, U. (2006) Rhythmic CLOCK-BMAL1 binding to multiple E-box motifs drives circadian Dbp transcription and chromatin transitions. Nat. Genet. 38, 369-374.

[38] Eissenberg, J.C. and Shilatifard, A. (2006) Leaving a mark: the many footprints of the elongating RNA polymerase II. Curr. Opin. Genet. Dev. 16, 184-190.

[39] Menet, J.S., Abruzzi, K.C., Desrochers, J., Rodriguez, J. and Rosbash, M. (2010) Dynamic PER repression mechanisms in the Drosophila circadian clock: from on-DNA to off-DNA. Genes Dev. 24, 358-367.

[40] Schmutz, I., Ripperger, J.A., Baeriswyl-Aebischer, S. and Albrecht, U. (2010) The mammalian clock component PERIOD2 coordinates circadian output by interaction with nuclear receptors. Genes Dev. 24, 345-357.

[41] Bozek, K., Relogio, A., Kielbasa, S.M., Heine, M., Dame, C., Kramer, A. and Herzel, H. (2009) Regulation of clock-controlled genes in mammals. PLoS One 4, e4882.

[42] Hendrich, B., Guy, J., Ramsahoye, B., Wilson, V.A. and Bird, A. (2001) Closely related proteins MBD2 and MBD3 play distinctive but interacting roles in mouse development. Genes Dev. 15, 710-723.

[43] Ng, H.H., Zhang, Y., Hendrich, B., Johnson, C.A., Turner, B.M., ErdjumentBromage, H., Tempst, P., Reinberg, D. and Bird, A. (1999) MBD2 is a transcriptional repressor belonging to the MeCP1 histone deacetylase complex. Nat. Genet. 23, 58-61.

[44] Sakamoto, Y., Watanabe, S., Ichimura, T., Kawasuji, M., Koseki, H., Baba, H. and Nakao, M. (2007) Overlapping roles of the methylated DNA-binding protein MBD1 and polycomb group proteins in transcriptional repression of HOXA genes and heterochromatin foci formation. J. Biol. Chem. 282, 16391-16400.

[45] Zhang, Y., Ng, H.H., Erdjument-Bromage, H., Tempst, P., Bird, A. and Reinberg, D. (1999) Analysis of the NuRD subunits reveals a histone deacetylase core complex and a connection with DNA methylation. Genes Dev. 13, 1924-1935.

[46] Zhang, F., Pomerantz, J.H., Sen, G., Palermo, A.T. and Blau, H.M. (2007) Active tissue-specific DNA demethylation conferred by somatic cell nuclei in stable heterokaryons. Proc. Natl. Acad. Sci. USA 104, 4395-4400.

[47] Bönsch, D., Hothorn, T., Krieglstein, C., Koch, M., Nehmer, C., Lenz, B., Reulbach, U., Kornhuber, J. and Bleich, S. (2007) Daily variations of homocysteine concentration may influence methylation of DNA in normal healthy individuals. Chronobiol. Int. 24, 315-326.

[48] Ji, Y., Qin, Y., Shu, H. and Li, X. (2010) Methylation analyses on promoters of mPer1, mPer2, and mCry1 during perinatal development. Biochem. Biophys. Res. Commun. 391, 1742-1747.
[49] Shih, M.C., Yeh, K.T., Tang, K.P., Chen, J.C. and Chang, J.G. (2006) Promoter methylation in circadian genes of endometrial cancers detected by methylation-specific PCR. Mol. Carcinog. 45, 732-740.

[50] Hsu, M.C., Huang, C.C., Choo, K.B. and Huang, C.J. (2007) Uncoupling of promoter methylation and expression of Period1 in cervical cancer cells. Biochem. Biophys. Res. Commun. 360, 257-262.

[51] Gery, S., Komatsu, N., Kawamata, N., Miller, C.W., Desmond, J., Virk, R.K. Marchevsky, A., McKenna, R., Taguchi, H. and Koeffler, H.P. (2007) Epigenetic silencing of the candidate tumor suppressor gene Per1 in non-small cell lung cancer. Clin. Cancer Res. 13, 1399-1404.

[52] Yang, M.Y., Chang, J.G., Lin, P.M., Tang, K.P., Chen, Y.H., Lin, H.Y., Liu, T.C., Hsiao, H.H., Liu, Y.C. and Lin, S.F. (2006) Downregulation of circadian clock genes in chronic myeloid leukemia: alternative methylation pattern of hPER3. Cancer Sci. 97, 1298-1307.

[53] Hoffman, A.E., Yi, C.H., Zheng, T., Stevens, R.G., Leaderer, D., Zhang, Y., Holford, T.R., Hansen, J., Paulson, J. and Zhu, Y. (2010) CLOCK in breast tumorigenesis: genetic, epigenetic, and transcriptional profiling analyses. Cancer Res. 70 1459-1468.

[54] Shi, Y., Lan, F., Matson, C., Mulligan, P., Whetstine, J.R., Cole, P.A. and Casero, R.A. (2004) Histone demethylation mediated by the nuclear amine oxidase homolog LSD1. Cell 119, 941-953.

[55] Wang, J., Scully, K., Zhu, X., Cai, L., Zhang, J., Prefontaine, G.G., Krones, A., Ohgi, K.A., Zhu, P., Garcia-Bassets, I., et al. (2007) Opposing LSD1 complexes function in developmental gene activation and repression programmes. Nature 446 , 882-887.

[56] Katoh, Y, and Katoh, M. (2007) Comparative integromics on JMJD2A, JMJD2B and JMJD2C: preferential expression of JMJD2C in undifferentiated ES cells. Int. J. Mol. Med. 20, 269-273.

[57] Takeuchi, T., Watanabe, Y., Takano-Shimizu, T. and Kondo, S. (2006) Roles of jumonji and jumonji family genes in chromatin regulation and development. Dev. Dyn. 235, 2449-2459.

[58] Tan, H., Wu, S., Wang, J. and Zhao, Z.K. (2008) The JMJD2 members of histone demethylase revisited. Mol. Biol. Rep. 35, 551-556.

[59] Lan, F., Bayliss, P.E., Rinn, J.L., Whetstine, J.R., Wang, J.K., Chen, S., Iwase, S. Alpatov, R., Issaeva, I., Canaani, E., et al. (2007) A histone H3 lysine 27 demethylase regulates animal posterior development. Nature 449,689-694.

[60] De Santa, F., Totaro, M.G., Prosperini, E., Notarbartolo, S., Testa, G. and Natoli, G. (2007) The histone H3 lysine-27 demethylase Jmjd3 links inflammation to inhibition of polycomb-mediated gene silencing. Cell 130, 1083-1094.

[61] Agger, K., Cloos, P.A., Christensen, J., Pasini, D., Rose, S., Rappsilber, J., Issaeva, I. Canaani, E. Salcini, A.E. and Helin, K. (2007) UTX and JMJD3 are histone H3K27 demethylases involved in HOX gene regulation and development. Nature 449 , $731-734$

[62] Barreto, G., Schafer, A., Marhold, J., Stach, D., Swaminathan, S.K., Handa, V., Doderlein, G., Maltry, N., Wu, W., Lyko, F., et al. (2007) Gadd45a promotes epigenetic gene activation by repair-mediated DNA demethylation. Nature $445,671-675$.

[63] Kriaucionis, S. and Heintz, N. (2009) The nuclear DNA base 5hydroxymethylcytosine is present in Purkinje neurons and the brain. Science 324, 929-930.

[64] Feng, D., Liu, T., Sun, Z., Bugge, A., Mullican, S.E., Alenghat, T., Liu, X.S. and Lazar, M.A. (2011) A circadian rhythm orchestrated by histone deacetylase 3 controls hepatic lipid metabolism. Science 331, 1315-1319. 